\title{
$\widehat{A}$ Madridge \\ madridge Journal of Clinical Research
}

Interconnecting Scientific World

Review Article

Open Access

\section{Proposed Prophylactic use of Lithium to improve Cognitive Decline and Mental Health in Disorders such as Alzheimer's Disease and Depression}

\author{
Jessica L Foster ${ }^{1}$ and Vincent Gallicchio ${ }^{2 \star}$ \\ ${ }^{\prime}$ Department of Health Science, College of Health and Human Development, Clemson University, USA \\ ${ }^{2}$ Department of Biological Sciences, College of Science, Clemson University, USA
}

\section{Article Info}

*Corresponding author:
Vincent Gallicchio
Professor
122 Long Hall
Department of Biological Sciences
College of Science
Clemson University
South Carolina, 29634
USA
E-mail: vsgall@clemson.edu

Received: January 2, 2018

Accepted: January 16, 2018

Published: January 22, 2018

Citation: Foster JL, Gallicchio VS. Proposed Prophylactic use of Lithium to improve Cognitive Decline and Mental Health in Disorders such as Alzheimer's Disease and Depression. Madridge J Clin Res. 2018; 2(1): 44-49.

doi: $10.18689 / \mathrm{mjcr}-1000108$

Copyright: @ 2018 The Author(s). This work is licensed under a Creative Commons Attribution 4.0 International License, which permits unrestricted use, distribution, and reproduction in any medium, provided the original work is properly cited.

Published by Madridge Publishers

\begin{abstract}
Background: Lithium is a naturally occurring element with many pharmacological applications, but less known is its potential use as a preventative or early treatment method for cognitive disorders such as Alzheimer's Disease (AD) and other mental health conditions.

Methods: An electronic search was conducted on Medline and PubMed through online research database systems, using the Keywords "lithium," "mental health," "bipolar disease," and "Alzheimer's disease." Searches for pertinent literature reviews and experimental studies took place between June 22, 2017 and December 20, 2017. 33 articles were referenced in the final product.
\end{abstract}

Results: Lithium interacts with cognitive processes and $A D$ pathology through reduction in Tau hyperphosphorylation and the formation of $A \beta$ plaques, reduction in the secretion of inflammatory cytokines from activated microglia, and promotion of neurogenesis through endogenous stem cell development. Lithium use also decreases cognitive deficits resulting from AD. Potential adverse effects of long-term lithium use can be minimized if proper precautions are taken, including the use of slow-release formulations and adjustment of serum levels as needed.

Conclusions: Lithium use clinically is a standard treatment protocol for bipolar disorder and its use is also indicated in association with other antidepressants for unipolar depression and potentially other mental health issues including those like AD that are associated with cognitive declines in memory. The short-term use of lithium clinically has only mild side effects and that the most severe side effects (directly on target organs) are not common and can be prevented through periodical monitoring in subjects requiring chronic treatment. Thus, it is proposed that prophylactic lithium may be effective as a prevented measure to restrict, inhibit or possibly even reverse conditions associated with cognitive declines in memory or related mental conditions.

Keywords: Alzheimer's disease; Lithium; Pluripotency markers; GSK-3 $\beta$; Phosphorylation.

\section{Introduction}

Lithium, a naturally occurring element, has a wide range of uses, including many industrial and pharmacological applications [1]. Lithium occurs naturally in soil and water, mostly at low concentrations, thus it enters the food chain [2]. It is still under debate whether lithium is an essential trace element; however, sufficient evidence exists showing lithium at low levels has beneficial effects on living organisms, especially with respect to normal physiology related to the reproductive system [3]. Lithium salts, particularly lithium carbonate, as a psychotropic drug, serves as a mood stabilizer in 
bipolar disorder patients receiving lithium as therapy. Of importance is the observation that in this cohort of patients, lithium use demonstrates the lowest relapse rate for the treatment of mania and depression than other comparable treatment options $[4,5]$. As such, a study conducted by the Institute of Medicine documented that as a treatment of choice for mood related disorders the overall impact of lithium in terms of reduced health care savings dollars was substantial in the millions of dollars [6]. In addition, importantly, lithium has been shown to decrease suicide rates in the bipolar patient population [4, 7]. At the same time, there are less than desirable effects associated with lithium clinically that must be mentioned. These include minor side effects such as increased thirst and urine output, fine hand tremors, nausea, appetite loss, diarrhea, sedation, and dizziness. More serious side effects associated with lithium use can be kidney problems and altered thyroid function [5]. A small cohort of patients taking lithium have also reported difficulty maintaining attention and concentration, however; it is hard to determine whether this symptom is due to their underlying mental disease or treatment [8]. Many of these unwanted side-effects can be attributed to lithium dosing, thus alternative lithium formulations such as slow-release lithium capsules combined with bedside monitoring of systemic lithium levels in blood significantly ameliorates these adverse conditions [9].

Psychological disorders have an undeniably large effect on the human brain and as such subsequently the many processes it regulates. For example, impairments in adult neurogenesis, neuroplasticity, and cellular resistance are all associated with bipolar disease [10]. As neurons in the human brain die, they go through extensive neurodegenerative changes, such as change in mitochondrial function and damage to dendrites, nerve terminals, myelin sheaths, and intracellular DNA. In addition, the synapses between neurons are lost, severely impacting neuronal communication [11]. The enzyme glycogen synthase kinase-3 (GSK-3) is closely associated with these neurodegenerative changes. GSK-3, made up of alpha- $\alpha$ and beta- $\beta$ counterparts, is a serine/threonine kinase that works through phosphorylation and has a role in the division, proliferation, differentiation, and adhesion of cells, among other functions $[1,12]$. GSK-3 is important physiologically for the normal functioning of the brain, due to its ability to eliminate excess neurons; however, GSK dysfunction is closely related to many neurodegenerative disorders, such as Parkinson's disease, type 2 diabetes, fragile $X$ syndrome, multiple sclerosis, and Alzheimer's Disease (AD) $[4,12]$.

Lithium is an inhibitor of GSK-3ß; in fact, it is the most effective inhibitor of the enzyme. It exerts this inhibition as the result of its ability to fit directly into a pocket in the GSK-3 $\beta$ protein, disabling GSK-3's phosphorylation action that ultimately contributes to neurodegeneration [4]. GSK-3 $\beta$ interacts with over 25 different transcription factors, including the inhibition transcription factors CREB (CAMP) response element-binding protein and $\beta$-catenin, both of which promote cell survival $[4,10]$. By inhibiting GSK-3 $\beta$, lithium allows these transcription factors, among others, to sustain function in cellular resistance, therefore opposing neurodegeneration. Transcription factor $\mathrm{pf} 3$, on the other hand, promotes cell death. It is activated by GSK- $3 \beta$ and as such can be inhibited by lithium. Other than its interaction with transcription factors, lithium's inhibition of GSK-3 $\beta$ promotes neuroprotection via a number of pathways such as through axonal regeneration and re-myelination, improved mitochondrial function, and neurogenesis in the hippocampal areas of the brain [4].

Alzheimer's disease (AD) is most prominent when characterizing neurodegenerative diseases and as such is the most common form of dementia. It was named the 6th leading cause of death in America in 2015 [13]. An estimated 5.5 million Americans of all ages have Alzheimer's disease. Of the estimated 5.5 million Americans living with Alzheimer's dementia in 2017, an estimated 5.3 million are age 65 and older and approximately 200,000 individuals are under age 65 and have younger- onset Alzheimer's. Collectively the calculated cost in terms of health care dollars is $\$ 259$ billion. The projected increases for new cases of Alzheimer's disease are expected to reach 16 million by the year 2050. One in three senior citizens in the country dies as the result of Alzheimer's disease and a new case is diagnosed every 30 seconds in the United States [14].

$A D$ is characterized by a buildup of amyloid plaques, caused by an accumulation of the amyloid-beta peptide $[4,5]$. In the brain of Alzheimer's patients amyloid plaques block the synapses between neurons. This results in the disruption of normal cell-to-cell signaling [13]. The amyloid-beta protein activates GSK-3, resulting in the hyperphosphorylation of Tau proteins in neurons $[5,13]$. GSK-3 $\beta$ is the primary enzyme that contributes to the hyperphosphorylation of Tau proteins [10]. The consequence of such abnormal phosphorylation is tangles of Tau proteins, referred to as "neurofibrillary tangles." Tau proteins are typically beneficial to the brain, aiding in the circulation of nutrients within brain cells, but neurofibrillary tangles disrupt this circulation and result in neuronal cell death [13]. Due to the important role GSK-3 $\beta$ plays in the progression of $A D$, one of the most promising potential uses of lithium and its inhibitory effect on GSK- $3 \beta$ is the prevention of AD. Many clinical and experimental trials on humans and animals alike have proved the effectiveness of lithium on dementia and $A D$, especially when treatment begins before the disease damages neurons beyond their point of repair [4].

\section{Discussion}

The pathology of $A D$ is very important to understand when considering prevention or treatment methods. Duncan et al. [15] placed the pathologic characteristics of AD into four major categories: 1) the hyperphosphorylation of Tau; 2 ) the aggregation of $A \beta$ proteins into amyloid plaques; 3 ) the production of inflammatory cytokines by activated microglia; and 4) significant neuronal and synaptic loss [15]. In addressing these pathological characteristics, lithium has been shown to counteract all of these pathologies, a finding that is very significant for the future of early AD treatment and prevention. 


\section{Hyperphosphorylation of Tau and Accumulation of $A \beta$ Plaques}

Lithium's ability to reduce Tau hyperphosphorylation and the formation of $A \beta$ plaques stands as one of the primary reasons why the drug serves as a great candidate for use in possible prevention and treatment of $A D$. A reduction in levels of Tau protein was indeed observed in a 2005 study on transgenic mice over expressing mutant human Tau, a mutation that causes the development of neurofibrillary tangles. After injecting experimental mice with $0.6 \mathrm{M}$ lithium chloride, at 10 micro liters per gram body weight, researchers observed reduced GSK-3 $\beta$ activity and reduced levels of insoluble Tau [16]. Further, in a 2014 study by Noble et al., [17], when the hindbrain ventricles of zebra fish embryos were injected with amyloid-beta peptide, Tau phosphorylation increased significantly and cognitive deficits were observed, as one might expect. Incubation with lithium chloride reversed the effects of the $A \beta$ peptide injection, indicating that lithium treatment is significant to reduce the accumulation of $A \beta$ and insoluble Tau. Lithium treatment also showed a positive effect on cognitive abilities, as the $A \beta$ injected animals treated with lithium had better responses to aversive stimuli than watertreated $A \beta$ injected animals [17].

In 2016, 4-month old APP-PS1 transgenic mice co expressing mutations in the amyloid precursor protein and the preselin 1 gene, the causative agent of most familial $A D$, were given 12 weeks of daily intraperitoneal lithium chloride injections $[11,18]$. Results showed a decrease in levels of $A \beta$ plaques in both the hippocampal and cortical areas of the brain. The authors highlighted the fact that lithium is an activator of the Wnt/ $\beta$-catenin signaling pathway, which has a significant role in neuro protection against injury from $A \beta$ peptides; GSK3- $\beta$ inhibits the Wnt/ $\beta$-catenin pathway. Due to the aforementioned mutations, the transgenic mice showed lower levels of $\beta$-catenin and higher levels of activated GSK3- $\beta$. Once treated with lithium, $\beta$-catenin levels were restored and levels of inactivated GSK3- $\beta$ increased, indicating reactivation of the Wnt/ $\beta$ - catenin pathway and an increase in neuro protection from $A \beta$ injury [18].

\section{Microglial Activation and Inflammation}

When observing inflammatory responses in the brains of the APP-PS1 transgenic mice, it was discovered that the lithium treatment reduced the inflammatory mechanisms of microglial activation [18]. This is a critical component to the use of lithium clinically for AD. Activated microglia, one of the core pathologies of $A D$ are central nervous system macrophages. They are especially present in the early stages of AD development and as such it may have great significance due to their production of inflammatory cytokines [15]. The inflammatory mechanisms of microglial activation have been shown to promote neurodegeneration in the early mild cognitive impairment stage of $A D$ as well as the early stages of Parkinson's disease (PD). Lithium treatment has been shown to counteract these neurodegenerative processes by down regulating proinflammatory genes whilst up regulating anti-inflammatory genes. Lithium has a higher therapeutic impact on microglial activation if treatment occurs early on in neurodegenerative disease due to the high numbers of proinflammatory mediators during early stages of disease [19].

A study by conducted Ferensztajn-Rochowiak et al., [20], to be published in 2018, evaluated the effects of long-term lithium treatment on concentrations of very- small embryoniclike stem cells (VSELs) and mRNA expression of pluripotency and glial markers in peripheral blood. Participants included 30 bipolar depression (BD) patients in remission and 15 healthy control subjects. Of the 30 BD patients, 15 had been treated continuously with lithium carbonate, with a mean treatment length of 16 years and serum concentration of $0.5-0.8 \mathrm{mmol} / \mathrm{l}$. Results showed that BD patients not taking lithium showed a significantly increased level of VSELs as compared to controls, while BD patients taking lithium showed a level of VSELS similar to controls. The number of VSELs in lithium treated BD patients was negatively correlated with the duration of lithium treatment and serum lithium concentration. In addition, among BD patients not treated with lithium, the mRNA levels of pluripotency markers, glial markers, and inflammatory cytokines were significantly higher than those of healthy controls [20].

VSELs can have a significant impact on the inflammatory mechanisms of $B D$ and $A D$ alike. Although VSELs have shown regenerative function in some organs, such as blood vessels and cardiac tissue, they can act erroneously, accumulating in areas of high inflammation and worsening negative pathologic effects. Results suggest that long- term lithium treatment normalizes the number of VSELs and reduces the negative effects produced by VSEL accumulation. Results also demonstrated that long-term lithium treatment has the ability to weaken the effect of the inflammatory processes, compensatory mechanisms, and regenerative responses that are associated with an increase in peripheral mRNA markers. Lithium produces these effects primarily through interactions with transcription factors Oct-4, Sox-2, GFAP, and Olig1 [20].

In summary, Ferensztajn-Rochowiak et al., [20], suggest that increased mobilization of VSELs, increased mRNA expression of pluripotency and glial cell markers, and elevation of inflammatory cytokines are all associated with repeated exposures to stress and the specific chronic inflammation that occurs during BD pathogenesis. Long-term lithium treatment was shown to have a significant protective effect on all of these factors. The neuroinflammation processes involved in $B D$ and $A D$ are very similar, both strongly associated with microglial activation. For this reason, lithium can be presumed to have the same effect on AD pathology. Therefore, lithium's ability to decrease levels of VSELs, pluripotency and glial cell markers, and inflammatory cytokines contributes to lithium's candidacy as a treatment method for AD [20].

\section{Stem Cells}

As was just mentioned lithium influences stem cells. The importance of stem cells in AD therapy is becoming increasingly recognized. Lithium therapy promotes endogenous stem cell development, making lithium a very useful drug for neurogenesis. The types of stem cells most commonly emphasized in $A D$ 
research are embryonic stem cells (ESCs), mesenchymal stem cells (MSCs), brain-derived neural stem cells (NSCs), induced pluripotent stem cells (iPSCs), and now very small embryonic stem cells (VSELs) [15, 20]. A systematic review published in 2015 by Ferensztajn-Rochowiak and Rybakowski [21] evaluated the effects of lithium on many different classes of stem cells. In this review, it was reported that lithium has the ability to maintain the pluripotency of MSCs, while concentrations less than $5 \mathrm{mmol} / \mathrm{l}$ promote MSC proliferation. This is important due to the many roles of MSCs in the pathology of AD. Rodent models of AD have shown that MSC transplantation can improve spatial learning and memory deficits, inhibit cell death from accumulation of $A \beta$ protein and Tau, reduce the formation of $A \beta$ plaques, reduce levels of inflammatory cytokines from activated microglia, and promote neurogenesis, synaptogenesis, and neuronal differentiation [15].

As demonstrated in rodent models, NSCs have also been shown to have a significantly positive impact on AD through the promotion of neurogenesis and cognitive function [15]. Lithium has been found to stimulate the self-renewal of neural progenitor cells, which serve as one of the two main sources of neurogenesis in the brain [21,22]. The drug is also reported to enhance the mitotic activity of Schwann cells and cause an increase in the presence BrdU labeled cells, used to detect proliferation of cells in brain tissues [22]. iPSCs have also shown great potential for use in AD treatment. When iPSCderived cholinergic neural precursors were transplanted into the hippocampus of a transgenic AD mouse model, these cells differentiated into mature cholinergic neurons and reversed spatial memory impairment. Additionally, iPSCs have been found to reduce the production of pro-inflammatory factors, improving neurological function in rodent models of ischemic stroke [15].

\section{Neuronal Loss}

The mass neuronal and synaptic loss that occurs in $A D$ pathology has the most observable effect on individuals, as the cognitive decline that occurs in early $A D$ is strongly associated with these occurrences [15]. Lithium stimulates neurogenesis in the brain, thus providing a protective effect from the neurodegeneration that occurs as $A D$ progresses. It has been found that lithium increases the volumes of grey matter in the brain, especially in the anterior cingulate and paralimbic cortical areas of the brain that have a large role in attention, motivation, and emotional modulation [10]. In $A D$, one of the largest areas of neuronal loss is the hippocampus; because of the hippocampus' large role in learning and memory, the amnestic symptoms that occur in the early stages of $A D$ can be attributed to loss in this part of the brain [15]. Lithium has the ability to stimulate hippocampal neurogenesis. A 2016 study evaluating the effects of lithium on hippocampal cells found that the injection of mice with a $2.5 \mathrm{~g} / \mathrm{kg}$ dose of lithium carbonate for four weeks resulted in an increase of cells in the dentate gyrus of the hippocampus [23].

$\mathrm{N}$-acetyl-aspartate (NAA) is a marker of neuronal viability and functionality. When the amount of NAA in the brain cortex of bipolar disease patients and a healthy control group were measured, it was found that NAA levels increased in both bipolar affected and non-affected participants when they were exposed to chronic lithium treatment [10, 11]. In another study, when 21 adults (12 of them bipolar patients) were placed on a 4-week lithium treatment regimen, increased NAA levels were also found. Additionally, in a study of healthy controls compared to bipolar patients taking lithium and valproate, it was shown that valproate did not significantly increase the cortex concentration of NAA; only lithium treated bipolar patients showed such an increase [11].

\section{Cognitive Deficits}

As mentioned previously, lithium has great potential to be used as a prevention method for $A D$, but is being most effective when applied before onset of the disease or in the very early stages of the disease. In 2011 Forlenza et al., [24] performed a randomized controlled trial to evaluate the progression of cognitive deficits in patients aged 60 years or older with amnestic mild cognitive impairment (aMCl). Individuals with aMCI have a high risk of developing dementia. Participants were put on a yearlong treatment regimen, given daily doses of $150 \mathrm{mg}$ lithium carbonate at serum levels of 0.25-0.5 mmol/l. Results showed a $50 \%$ reduction in GSK-3 activity in leukocytes and a reduction in cognitive decline when compared to the control group taking the placebo. The yearlong lithium treatment program also decreased levels of Tau-phosphorylation, one of the major precursors to $A D$, in the cerebrospinal fluid of the individuals who did not later develop AD. Perhaps the most important conclusion that can be taken from this study is that the use of lithium for prevention of $A D$ is likely stage-dependent [24].

A 2015 study on transgenic mice exhibiting a mousemodel of Alzheimer's disease placed mice on a $1.5-\mathrm{mg} /$ day micro dose of lithium carbonate for 8 or 16 months. The results of this study showed that anxiety and spatial and aversiverelated memory deficits resulting from the neurodegenerative processes of $A D$ could be prevented by lithium treatment. In addition, lithium was found to reduce density of amyloid plaques and protect mice from hippocampal apoptosis, while increasing neuronal density in the prefrontal cortex. An increase was also seen in BDNF (brain derived neurotrophic factor) density, which is important due to BDNFs role in neuronal communication [25]. This was not the first time that lithium's effect on BDNF levels were reported; in 2009 Leyhe et al., conducted a 10-week trial on $A D$ patients in order to evaluate the influence of lithium treatment on BDNF serum concentration. BDNF is an especially important growth factor due to its role in CNS synaptic plasticity as well as neuronal survival and differentiation. All of the $A D$ patients at the beginning of the trial had low levels of BDNF, but after 10 weeks of being administered lithium sulfate at a serum level of 0.4-0.8 $\mathrm{mmol} / \mathrm{L}$, a significant increase in BDNF concentration was observed. The patients also showed a decrease in cognitive decline, as can be seen in many other similar trials [26].

When bipolar patients' cognitive functioning was evaluated by their performance on the Cambridge Neuropsychological Test Automated Battery (CANTAB), it was found that all $\mathrm{BD}$ patients had decreased memory and 
executive function ability when compared to healthy controls; however only BD patients not taking lithium had visual memory impairments. Due to the hippocampus' role in visual memory, it can be hypothesized from the results of this study that lithium may have a neuroprotective effect on the hippocampus [27]. The memory deterioration that occurs during $A D$ is also due in part to cholinergic dysfunction. In a study aimed to evaluate the relationship between cholinergic dysfunction and lithium, rats were given an intraperitoneal injection of scopolamine to create a mouse model of cholinergic dysfunction. Upon injection, activation of GSK-3 occurred, causing spatial learning problems and memory deficits in the rats. However, if the intraperitoneal injection was pretreated with lithium, those deficits were prevented [28].

\section{Incidence of AD in Individuals Exposed to Lithium}

In addition to the many trials that demonstrate the effect of lithium on the brain and cognitive functioning in $B D$ and $A D$, there have been numerous studies directly observing the incidence of dementia and/or Alzheimer's in individuals exposed to lithium. In a study published in the British Journal of Psychology, Gerhard et al., evaluated the correlation between lithium treatment and risk of dementia directly through an observational cohort study on BD diagnosed individuals over 50 years of age. They found that continuous lithium use, defined as 301-365 days of lithium medication supply in one year, was associated with a decreased dementia incidence rate [29]. A similar Brazilian study by Nunes et al., [30], compared 66 elderly BD patients (60 years of age or older) treated with lithium therapy to 48 elderly BD patients not on recent lithium therapy. Results showed a $5 \%$ prevalence of $A D$ in the lithium-treated group and a $33 \%$ prevalence of $A D$ in the group of patients who had not been given lithium therapy. The expected rate of $A D$ in the general Brazilian population was $7 \%$; the similar prevalence in the lithium treatment group (5\%) indicates that lithium treatment can reduce risk of $A D$ in $B D$ patients to the risk level of the general population [30].

Interestingly, a 2017 Danish population-based case control study looking at patients with dementia as well as non-dementia patients discovered an association between dementia and lithium in public drinking water. Lithium levels were estimated using samples from 151 public waterworks that supplied water to $42 \%$ of the country. Results showed that healthy controls without a dementia diagnosis were more likely to have higher levels of lithium in their drinking water when compared to the level of lithium in the water of dementia patients. Specifically, those test subjects who had lithium levels of 10.1 to 15.0 micro/L or greater than 15.0 micro/L in their drinking water had lower rates of dementia. The same general conclusion was found when the researchers examined the relationship between lithium and drinking water in Alzheimer's disease and vascular dementia [31].

While lithium is a very popular medication for BD patients, it is not the only option; anticonvulsants, antidepressants and antipsychotics are also popular forms of treatment. Out of these four options, however, lithium is the only one that has shown neuroprotective effects. A 2010 Danish study that included bipolar patients taking all four of these types of medications determined after a 10-year follow up that long-term lithium treatment was the only one of the four treatment options that resulted in a reduced rate of dementia later in life [32].

\section{Lithium Safety and Tolerability}

It is important to keep in mind the safety and tolerability of lithium treatment. According to a 2014 review by Aprahamian et al., [33], lithium treatment can result in various adverse symptoms, such as abdominal discomfort, diarrhea, tremor, weight gain, edema, polyuria, nocturia, and erythema. These symptoms, especially those involving the digestive system, can be decreased by the use of a slow-release form of the drug or lithium citrate as a substitution for lithium carbonate. It is well known that lithium treatment can cause thyroid dysfunction through the inhibition of thyroid hormone production; therefore, it is important to evaluate a patient's thyroid function prior to the beginning of lithium treatment, after 3 months, and every 6-12 months during the entire course of treatment. Levels of thyroid stimulating hormone (TSH), free thyroxine, parathyroid hormone (PTH), and calcium should be monitored over the course of treatment to check for conditions such as hyperthyroidism, hypothyroidism, hyperparathyroidism, and hypercalcemia. Another possible adverse effect of lithium treatment includes renal impairment, in the form of tubular and glomerular dysfunction indicated by a reduction in renal filtration capacity and decreased urinary concentration, seen in 50\% of patients prescribed lithium. To avoid severe and irreversible consequences of tubular impairment, such as diabetes insipidus, simple urinary analyses should be utilized. Additionally, structural changes in the kidneys, such as such as tubular atrophy and interstitial fibrosis, have been observed in some lithium users. It is important to monitor renal function in all patients prescribed lithium, but extra precaution should be taken in patients with high risk of renal dysfunction, such as patients previously diagnosed with hypertension or diabetes. It is also recommended that women discontinue lithium use in the first trimester of pregnancy, due to the risk of lithium being teratogenic to the developing child [33].

Regarding lithium's interactions with other drugs, Aprahamian et al., [33], reported drugs such as alprazolam, thiazide diuretics, ACE inhibitors, and anti- inflammatory nonsteroidal drugs increase the plasma concentrations of lithium. This creates a risk of lithium toxicity unless levels of lithium are reduced prior to treatment with one of the aforementioned drugs (i.e., reducing lithium levels by $40-50 \%$ prior to treatment with a diuretic). Conversely, theophylline, acetazolamide, mannitol and caffeine lower lithium concentrations by increasing clearance. Calcium channel antagonists and angiotensin II antagonists can be toxic and dangerous in combination with lithium treatment. Although there are adverse effects associated with lithium treatment, if medical personnel and patients alike are aware of the risks and take the appropriate precautionary measures, significant health risks can be minimized [33]. 


\section{Conclusion}

The body of evidence supporting the clinical use of lithium for $A D$ prevention and treatment are too numerous to ignore. Lithium treatment has been shown to reduce Tau hyperphosphorylation and the formation of $A \beta$ plaques, reduce the secretion of inflammatory cytokines from activated microglia, promote neurogenesis through endogenous stem cell development, and decrease cognitive deficits associated with $A D$. Potentially harmful effects of lithium treatment can be minimized if the appropriate precautions are taken. With the established costsavings of lithium use in terms of health expenditures directed to mental health and other related illnesses characterized by cognitive decline where this question was addressed, prophylactic lithium use as a preventive measure, defined as "low dose" are suggested. Defining clinical use of lithium in this context is therefore justified and warranted.

\section{Conflict of interest}

The authors of the present manuscript report no conflict of interest in the conduction of this study.

\section{References}

1. Gallicchio VS. Lithium as a Trace Element - Effects on Stem Cells. Trace Elements edited by F. Atroshi \& Tuomas Westernmarck. InTech Europe. 2018.

2. Inayat $\mathrm{M}$, Gallicchio VS. The effects of lithium on the immune system. In: Lithium in Neuropsychiatry, eds M. Bauer, P. Grof, B. Muller-Oerlinghausen, Informa Healthcare. United Kingdom. 2006; 1-532.

3. Bach RO, Gallicchio VS. Lithium and Cell Physiology. Springer. 1990; 1-174.

4. Jope RS, Nemeroff CB. Lithium to the Rescue. Cerebrum. 2016.

5. Duvall $A E$, Gallicchio VS. Lithium treatment in clinical medicine: history, current status, and future use. Journal of Cell Science and Therapy. 2017; 8(3): 1-8. doi: 10.4172/2157-7013. 1000270

6. Watt RJ, Henter IO, Jaminson JC. Lithium revisited: savings brought about by the use of lithium, 1970-1991. Psychiatr Q. 2001; 72(2): 149-166.

7. Saunders KEA, Cipriani A, Rendell J, et al. Oxford Lithium Trial of the early affective, cognitive, neural, and biochemical effects of lithium carbonate in bipolar disorder: study protocol for a randomized controlled trial. Trials. 2016; 17(116): 1-5. doi: 10.1186/s13063-016-1230-7

8. Malhi GS, Outhred T. Lithium Therapy, Bipolar Disorder- and Neurocognition. Psychiatric Times. 2017; 34(1).

9. Berghofer $A$, Grof $P$, Oerlinghausen BM. Recommendations for the safe use of lithium. In: Lithium in Neuropsychiatry, eds M. Bauer, P. Grof, B. Muller- Oerlinghausen. InformaHealthcare, United Kingdom. 2006; 1-532.

10. Dell'Osso L, Del Grande C, Gesi C, Carmassi C, Musetti C. A new look at an old drug: neuroprotective effects and therapeutic potentials of lithium salts. Neuropsychiatric Disease and Treatment. 2016; 12: 1687-1703. doi: 10.2147/NDT.S106479

11. Young W. Review of Lithium Effects on Brain and Blood. Cell Transplantation. 2009; 18(9): 951-975. doi: 10.3727/096368909X471251

12. Lei $P$, Ayton S, Bush A, Adlard PA. GSK-3 in Neurodegenerative Diseases. International Journal of Alzheimer's Disease. 2011. doi: 10.4061/2011/189246

13. Greenblat J, Grossman K. Lithium: The Cinderella Story about a Mineral That May Prevent Alzheimer's Disease. 2016

14. Latest Alzheimer's Facts and Figures. 2016.

15. Duncan T, Valenzuela M. Alzheimer's disease, dementia, and stem cell therapy. Stem Cell Research and Therapy. 2017; 8(1): 1-9. doi: 10.1186/ s13287-017-0567-5
16. Noble W, Planel E, Zehr C, et al. Inhibition of glycogen synthase kinase-3 by lithium correlates with reduced tauopathy and degeneration in vivo. PNAS. 2005; 102(19): 6990-6995. doi: 10.1073/pnas.0500466102

17. Nery LR, Eltz NS, Hackman C, et al. Brain Intraventricular Injection of Amyloid-B in Zebrafish Embryo Impairs Cognition and Increases Tau Phosphorylation, Effects Reversed by Lithium. PLoS One. 2014; 9(9). doi: 10.1371/journal.pone.0105862

18. Toledo EM, Inestrosa NC. Activation of Wnt signaling by lithium and rosiglitazone reduced spatial memory impairment and neurodegeneration in brains of an APPswe/PSEN1DE9 mouse model of Alzheimer's disease. Molecular Psychiatry. 2010; 15: 272-285. doi: 10.1038/mp.2009.72.

19. Lauterbach EC. Repurposing psychiatric medicines to target activated microglia in anxious mild cognitive impairment and early Parkinson's disease. American Journal of Neurodegenerative Disease. 2016; 5(1): 29-51.

20. Ferensztajn-Rochowiak E, Kucharska-Mazur J, Tarnowski M, et al. Stem cells, pluripotency and glial markers in peripheral blood of bipolar patients on long term lithium treatment. Progress in Neuro-psychopharmacology \& Biological Psychiatry. 2018; 80: 28-33. doi: 10.1016/j.pnpbp.2017.06.013

21. Ferensztajn-Rochowiak $E$, Rybakowski JK. The effect of lithium on hematopoietic, mesenchymal, and neural stem cells. Pharmacological Reports. 2016; 68(2): 224-230. doi: 10.1016/j.pharep.2015.09.005

22. Zhu Z, Yin J, Guan J, et al. Lithium stimulates human bone marrow derived mesenchymal stem cell proliferation through GSK-3b-dependent bcatenin/Wnt pathway activation. FEBS Journal. 2014; 281(23): 5371-5389. doi: $10.1111 /$ febs.13081

23. Rajkowska G, Clarke G, Mahajan G, et al. Differential effect of lithium on cell number in the hippocampus and prefrontal cortex in adult mice: a stereological study. Bipolar Disorder. 2016; 18(1): 41-51. doi: 10.1111/ bdi. 12364

24. Forlenza OV, Diniz BS, Radanovic M, et al. Disease- modifying properties of long-term lithium treatment for amnestic mild cognitive impairment: randomised control trial. The British Jounal of Psychiatry. 2011; 198(5): 351-356. doi: 10.1192/bjp.bp.110.080044

25. Nunes MA, Schöwe NM, Monteiro-Silva KC, et al. Chronic Microdose Lithium Treatment Prevented Memory Loss and Neurohistopathological Changes in a Transgenic Mouse Model of Alzheimer's Disease. PLOS ONE. 2015; 10(12). doi: 10.1371/journal.pone.0142267

26. Leyhe T, Eschweiler GW, Stransky E, et al. Increase of BDNF Serum Concentration in Lithium Treated Patients with Early Alzheimer's Disease. Journal of Alzheimer's Disease. 2009; 16(3): 649-656. doi: 10.3233/JAD2009-1004

27. Bersani G, Quartini A, Zullo D, lannitelli A. Potential neuroprotective effect of lithium in bipolar patients evaluated by neuropsychological assessment: preliminary results. Human Psychopharmacology Clinical Experimental. 2016; 31(1): 19-28. doi: 10.1002/hup.2510

28. Yuan-Yuan $W$, Xiong $W, L u T$, et al. Lithium Attenuates ScopalmineInduced Memory Deficits with Inhibition of GSK-3B and Preservation of Postsynaptic Components. Journal of Alzheimer's Disease. 2013; 37(3): 515-527. doi: 10.3233/JAD-130521

29. Gerhard T, Devanand DP, Huang C, Crystal S, Olfson M. Lithium treatment and risk for dementia in adults with bipolar disorder: population-based cohort study. The British Journal of Psychiatry. 2015; 207(1): 46-51. doi: 10.1192/bjp.bp.114.154047

30. Nunes PV, Forlenza OV, Gattaz WF. Lithium and risk for Alzheimer's disease in elderly patients with bipolar disorder. The British Journal of Psychiatry. 2007; 190(4): 359-360. doi: 10.1192/bjp.bp.106.029868

31. Kessing LV, Gerds TA, Knudsen NN, et al. Association of Lithium in Drinking Water with the Incidence of Dementia. JAMA Psychiatry. 2017; 74(10): 1005-1010. doi: 10.1001/jamapsychiatry.2017.2362

32. Kessing LV, Forman JL, Anderson PK. Does lithium protect against Dementia? Bipolar Disorders. 2010; 12(1): 87-94. doi: 10.1111/j.1399-5618.2009.00788.x

33. Aprahamian I, Teixeira de Sousa R, Valiengo LD, et al. Lithium safety and tolerability in mood disorders: a critical review. Rev Psiq Clin. 2014; 41(1): 9-14. doi: 10.1590/0101-60830000411914 